\title{
Pulsed Thermography applied to multi-material and multi-layer pomological models
}

\author{
by E. Grifoni*, J. Melada*, L. Bonizzoni*, M. Gargano*, I. Mignani**, N. G. Ludwig* \\ * Physics Department Aldo Pontremoli, University of Milan (UNIMI), via Celoria 16, Milan. \\ ${ }^{* *}$ Faculty of Agricultural and Food Sciences, University of Milan (UNIMI), via Celoria 2, Milan.
}

\begin{abstract}
Pulsed Thermography (PT) proves to be particularly suitable for the analysis of artworks realized with particularly heat-sensitive materials. The analytical integration of PT and other Imaging techniques (High Resolution Technical Photography and High Resolution Digital X-Radiography) provide information from the superficial, sub-superficial and innermost layers of the object, respectively, capturing both accurate spectral, spatial and density data. The paper presents a case study in which PT proves to be a highly predictive technique able to prevent or limit the loss of pictorial film in multimaterial and multi-layer artifacts such as pomological models.
\end{abstract}

\section{Introduction}

Active Infrared Thermography (a-IRT) is an imaging technique notoriously appreciated in the wide field of Cultural Heritage science $(\mathrm{CH})$ thanks to its ease of use, high thermal resolution and non-invasiveness. From the 1960 s to the present, several studies attest to its extreme versatility in the inspection of a variety of historical-artistic materials [1-3] and their respective decay phenomena in order to determine their preservation state. A-IRT involves the use of an external source to thermally stimulate the target and to highlight the regions of interest (thermal anomalies). Various methods and relative configurations exist for the acquisition of data through a-IRT. Those mainly applied to Conservation studies and $\mathrm{CH}$ are Pulsed Thermography (PT) and Lock-in Thermography (LT) exploited using optical thermal stimulation (e.g. flash lamps, incandescent lamps, quartz lamps) [4-5]. In the case of artworks realized with particularly heat-sensitive materials, protracted exposure may be highly discouraged. In order to prevent any further damage, PT is the most appropriate among the different active thermographic configurations for those distinctive artworks typology referred as ceroplastic. While PT highlights the presence, the extent and the depth of the sub-surface anomalies and discontinuities, High Resolution Technical Photography (Hi-ResTP) and High Resolution Digital X-Radiography (Hi-Res DXR) provide information from the superficial and innermost layers of the object, respectively, capturing both accurate spectral, spatial and density data. The analytical integration of the above-mentioned imaging techniques was essential to obtain a great richness of the informative contents; the optimization of the multimodal and multilayer data capturing strategy has enhanced the potential insights into data analysis of particularly complex artworks such as those examined in this research project.

\subsection{The Garnier Valletti Collection}

The pilot study here reported focuses on two artifacts belonging to the Garnier Valletti Collection of the University of Milan (UNIMI). This collection gathers about 1700 naturalistic models of fruits representing the many taxonomic lines of the European fruit germplasm that existed until the mid-nineteenth century. The models realized by the famous ceroplast Garnier Valletti are incredibly realistic as they exactly reproduce not only the shape and color but also the weight of the original fruits. They preserve a high cultural value as they emulate varieties now extinct from our current fruits production as well as a historical and artistic value for the excellent technical quality reached. The manufacturing technique is not entirely well known as the artist always wanted to jealously keep the secret of his recipes. Thanks to some manuscript documents, we have partial information on the use of materials such as wax, Dammar resin, etc. Other modelers, such as Dürfeld, used papier-mâché covered with gypsum and wax. These artifacts are therefore very complex multilayered systems whose analytical study is particularly challenging. This implies a holistic approach and a considerable effort in their chemical-physical characterization and interpretation, as well as substantial problems of conservation, restoration and transmission to the future. From this huge collection two exemplars have been selected, one referring to Garnier Valletti (apple Rome Beauty) (Fig. 1) and the other to Dürfeld (pear Britanische Herbstbirne) (Fig. 2), as they present two different preservation states even though they have always been kept in the same micro-environmental conditions: the first almost intact and the second with conspicuous swellings and falls of the pictorial film.

\section{Material and Methods}

The analytical protocol provides for the following techniques: Visible Fluorescence Induced by Ultraviolet radiation (UVF) (Figg. 3-4), Near-Infrared reflectography (NIR) (Figg. 5-6), Radiography (RX) (Figg. 7-8) and Pulse-Thermography (PT) (Figg. 9-10). For the first two Hi-Res Imaging techniques, the same PhaseOne XF IQ4 100 MP camera system, with a full 
frame medium format CMOS sensor modified to extend the sensitivity from $350 \mathrm{~nm}$ to $1000 \mathrm{~nm}$, was used in order to achieve 16-bit raw images of $8708 \times 11608$ pixels. As far as the XR is concerned, the portable and real-time Hi-Res CR scanner Dürr HD-CR-35ndt, with $25 \mu \mathrm{m}$ of spatial resolution, was chosen using a Gilardoni Artgil radiographic tube (80 kV max, 5 $\mathrm{mA}$ ) and imaging plates of $30 \times 40 \mathrm{~cm}$ in size. The PT inspection was exploited using an Avio R500EX-Pro (8-14 $\mu \mathrm{m}$ spectral range, sensitivity of $0.025^{\circ} \mathrm{C}$ at $30^{\circ} \mathrm{C}$ and $0.87 \mathrm{mrad}$ of spatial resolution) with a framerate of $30 \mathrm{~Hz}$. Two Godox WITSTRO AD360 flashes were used for the thermal stimulation of the target. The micro-detachments of the external pictorial film of the objects were evaluated analyzing the temperature evolution registered by the a-IRT. In order to highlight their localization an algorithm based on the fourth standardized central moment (kurtosis) was used [6-8].

\section{Figures}
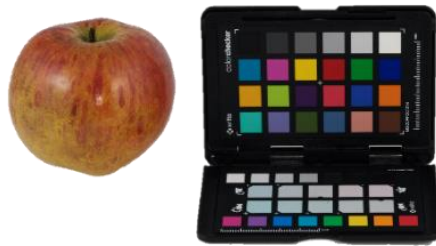

Fig. 1. Calibrated Visible image. Garnier Valletti (GV), apple Rome Beauty.
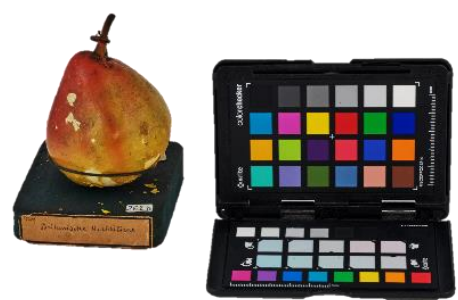

Fig. 2. Calibrated Visible image. Dürfled (Dü), pear Britanische Herbstbirne.

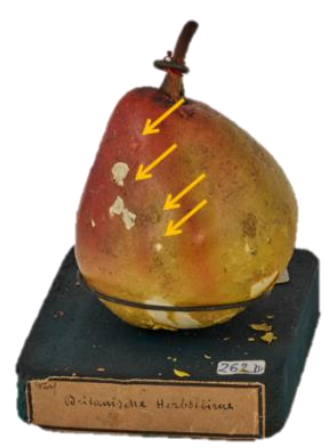

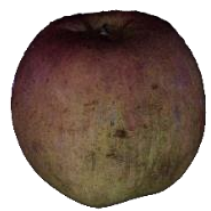

Fig. 3. UVF image. (GV) apple.

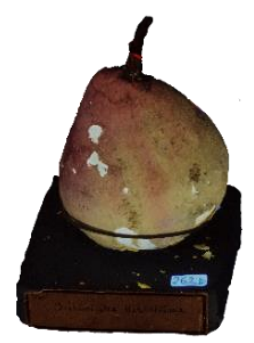

Fig. 4. UVF image. (Dü) pear.

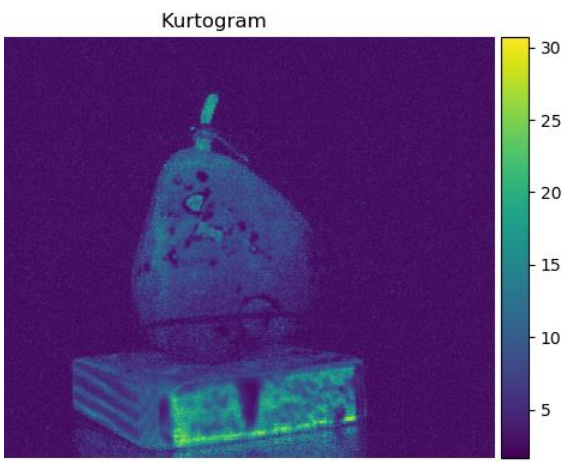

Fig. 9. PT image.

(Dü) pear.
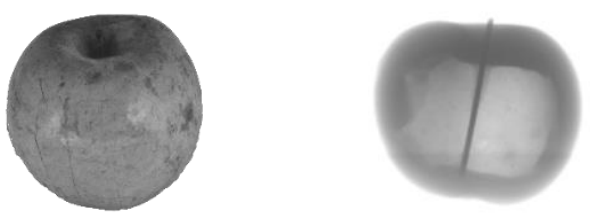

Fig. 5. NIR image. (GV) apple.

Fig. 7. $R X$ image. (GV) apple.

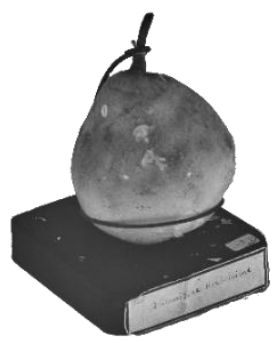

Fig. 6. NIR image. (Dü) pear.

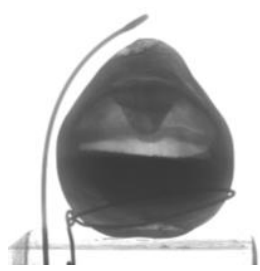

Fig. 8. $R X$ image. (Dü) pear.

\section{REFERENCES}

[1] Mercuri, F., Orazi, N., Paoloni, S., Cicero, C., \& Zammit, U. (2017). Pulsed thermography applied to the study of cultural heritage. Applied Sciences, 7(10), 1010.

[2] Ibarra-Castanedo, C., Sfarra, S., Ambrosini, D., Paoletti, D., Bendada, A., \& Maldague, X. (2010). Diagnostics of panel paintings using holographic interferometry and pulsed thermography. Quantitative InfraRed Thermography Journal, 7(1), 85-114.

[3] Bison, P., Clarelli, F., \& Vannozzi, A. (2015). Pulsed thermography for depth profiling in marble sulfation. International Journal of Thermophysics, 36(5-6), 1123-1130. 